\title{
Bohmian Trajectories of the Time-oscillating Schrödinger Equations
}

\author{
DANDAN Li*,1, JinqiaO DUAN ${ }^{2}$, Li Lin $^{1}$ AND Ao Zhang ${ }^{1}$ \\ 1. School of Mathematics and Statistics and Center for Mathematical Sciences, \\ Huazhong University of Science and Technology, Wuhan, 430074, PR China \\ 2. Departments of Applied Mathematics \& Physics, Illinois Institute of \\ Technology, Chicago, Illinois, 60616, USA
}

\begin{abstract}
Bohmian mechanics is a non-relativistic quantum theory based on a particle approach. In this paper we study the Schrödinger equation with rapidly oscillating potential and the associated Bohmian trajectory. We prove that the corresponding Bohmian trajectory converges locally in measure, and the limit coincides with the Bohmian trajectory for the effective Schrödinger equation on a finite time interval. This is beneficial for the efficient simulation of the Bohmian trajectories in oscillating potential fields.
\end{abstract}

॥

\section{Lead Paragraph}

Bohmian mechanics is a quantum theory of motion for concerning particles. Each particle has a position at all times and the evolution of these positions is governed by the usual quantum wave function which satisfies the Schrödinger equation. Roughly speaking, according to the theory of Bohmian mechanics the trajectories of electrons around atoms can be pictured as the motion of the solar system. The Schrödinger equation with rapidly oscillating potential arises in many fields. It has been shown that the large scale Schrödinger equation converges to an effective Schrödinger equation with averaged or homogenized potential under certain assumptions. A natural question is whether the corresponding Bohmian trajectory converges to the one of the effective Schrödinger equation. Due to the complicated relation between wave functions and the Bohmian trajectories, the convergence of the Schrödinger equations does not directly imply convergence under the corresponding Bohmian trajectories. The convergence of Bohmian measure can be obtained from convergence of wave function, and then combined with the properties of Young measure corresponding to Bohmian flow, the convergence of Bohmian trajectory in measure is obtained.

\section{Introduction}

Bohmian mechanics is a Galilei-invariant theory for the motion of particles ([ $[$ ] $)$. The theory was developed by Bohm in 1952 as an alternative explanation of the motion for particles in quantum mechanics. Mathematically, the law of motion is a first order differential equation and thus Bohmian mechanics is naturally formulated in configuration space. The configuration space of $N$ particles is the vector

$$
q=\left(q_{1}, \cdots, q_{N}\right) \in \mathbb{R}^{3 N},
$$

AMS Subject Classification: 35B27; 35J10; 35R06

${ }^{*}$ Corresponding author

Emails: dandanli@hust.edu.cn (D. Li); duan@iit.edu (J. Duan); linli@hust.edu.cn (L. Lin);

zhangao1993@hust.edu.cn (A. Zhang)

Keywords: Quantum mechanics; Schrödinger equation; Oscillating potential; Bohmian trajectories; Bohmian measure. 
where $q_{j} \in \mathbb{R}^{3}$ is the position of $j$-th particle. Given an initial state, the theory yields a trajectory

$$
t \rightarrow X(t) \in \mathbb{R}^{3 N},
$$

in configuration space, i.e. the position of particles at all time.

The positions of the particles can be obtained from Bohmian mechanics, and then velocities, momentum, energies and spin can be found through the positions ([3, 24, 27]). The law of motion for $X(t) \in \mathbb{R}^{N}$ is

$$
\frac{\mathrm{d}}{\mathrm{d} t} X(t)=\frac{\operatorname{Im}(\bar{\psi}(t, X(t)) \nabla \psi(t, X(t)))}{|\psi(t, X(t))|^{2}}
$$

where the wave function $\psi(t, x): \mathbb{R} \times \mathbb{R}^{3 N} \rightarrow \mathbb{C}$ solves the Schrödinger equation with a Hamiltonian operator $H$

$$
i \frac{\partial}{\partial t} \psi(t, x)=H \psi(t, x) \text {. }
$$

The wave function guarantees a time-dependent vector-field on configuration space. Its integral curves are the possible trajectories of Bohmian particles. Given initial data $X(0)$ and $\psi(0)$, the position $X(t)$ is determined for all time. The mathematical foundation of (1.1) is solid and the well-posedness (in the sense of some measure) can be found in 28] regardless of the continuity of the velocity field.

In this paper, we study the Bohmian trajectory corresponding to the large scale Schrödinger equation under rapidly oscillating potential. In recent years, the Schrödinger equation with rapidly oscillating potential function and rapidly oscillating force function has been extensively studied as a fundamental equation in modern mathematical physics. The large scale Schrödinger equation can be derived from plasma physics and as an amplitude equation in a perturbation study of sine-Gordon equation ([5]). The problem that involved with periodic coefficient was investigated in [4] and time-dependent periodic potential with large scale has been studied intensely in 1, 25]. Meanwhile, since the importance of incorporating stochastic effects in the modeling of complex systems has been recognized, the oscillating stochastic Schrödinger equation had also attracted a lot of attention $([10,21])$. It has been shown that the large scale Schrödinger equation converges to an effective Schrödinger equation with averaged or homogenized potential under certain assumptions. A natural question is whether the corresponding Bohmian trajectory converges to the one of the effective Schrödinger equation.

The study of the Bohmian trajectories arises from the semiclassically scaled Schrödinger equations ([26]). For the scaled Schrödinger equation, the limit of the wave functions and the corresponding Bohmian trajectories had been studied intensely. Note that nothing can yield by simply passing the scale parameter to 0, the classical limit of the scaled Schrödinger equation falls into the so called "singular limits" in asymptotic approximation theory ([13, 19, 20]) which has been studied in a variety of differential equations. However, as for the convergence of Bohmian trajectories, since the relation between the corresponding Bohmian trajectories and wave functions is quite complicated, it is very difficult to prove the convergence of Bohmian trajectories directly through the results of wave functions. In recent years, the study of the classical limit of Bohmian trajectories is of great interest. For wave packets as defined by Hagedorn, it was shown in [11] that the Bohmian trajectories converge to Newtonian trajectories in probability. Instead of studying Bohmian trajectories directly, [23] considered a class of phase space measure, i.e. Bohmian measure, which naturally arises in the Bohmian interpretation of quantum mechanics. The authors of [14] assumed that the initial data for the scaled Schrödinger equation were WKB (after three papers by Wentzel, Kramers and Brillouin respectively, in 1926) 
and used the theory of Young measure as a connection between the Bohmian flow and its limit. The analysis of oscillation and concentration effects in [14] and [23] was in the semi-classical regime.

Thus, the results in [11, 14, 23] and the study of convergence of the Schrödinger equation with rapidly oscillating functions provide us with a possibility to prove the convergence of corresponding Bohmian trajectory. In this paper, the convergence of the wave functions governed by the time-oscillating Schrödinger equations is proved under certain assumptions. This is then to consider the limit of the corresponding Bohmian trajectories. We start by deriving the limit of Bohmian measures in dependence on the scale of oscilations and concentrations of the sequence of wave functions under consideration. Furthermore, given the equivalence between Bohmian measure and the Bohmian flow, we also prove rigorously that the corresponding Bohmian trajectory converges locally in measure on the finite time interval, and the limit trajectory coincides with the effective system of the time-oscillating Schrödinger equation.

The rest of the paper is organized as follows. Section 2 is devoted to the basic setting of our problem and some preliminary results in oscillating periodic functions, measure theory, whereas in Section 3 the convergence theorems (Theorem 3.3 and Theorem 3.6) are established for both wave function satisfying the time-oscillating Schrödinger equation and the associated Bohmian trajectory. Finally, Section 4 is the Conclusion and Discussion section.

\section{Preliminaries}

Consider the wave function $\psi^{\varepsilon}(t, \cdot)$ in $\mathbb{R}^{N}$ satisfying the following time-oscillating Schrödinger equation:

$$
\left\{\begin{array}{l}
i \partial_{t} \psi^{\varepsilon}(t, x)=-\frac{1}{2} \Delta \psi^{\varepsilon}(t, x)+V\left(\frac{t}{\varepsilon}, x\right) \psi^{\varepsilon}(t, x), \quad x \in \mathbb{R}^{N}, t>0, \\
\left.\psi^{\varepsilon}(t, x)\right|_{t=0}=\psi_{0}^{\varepsilon},
\end{array}\right.
$$

where $\psi_{0}^{\varepsilon} \in H^{1}\left(\mathbb{R}^{N}\right), N \geq 3, \varepsilon>0$ and the Hamiltonian operator $H=-\frac{1}{2} \Delta+V(\cdot, \cdot)$.

The potential $V(t, x) \in C_{0}^{\infty}\left(\mathbb{R} \times \mathbb{R}^{N} ; \mathbb{R}\right)$ with period 1 is assumed to be bounded below and subquadratic, i.e.,

$$
\partial_{x}^{k} V(\cdot, x) \in L^{\infty}\left(\mathbb{R}^{N}\right) \quad \forall k \in \mathbb{N}^{n} \text { such that }|k| \geq 2 .
$$

Let $V^{\star}(x):=\int_{0}^{1} V(t, x) \mathrm{d} t$, the effective system of the time-oscillating Schrödinger equation (2.1) is written as

$$
\left\{\begin{array}{l}
i \partial_{t} \psi(t, x)=-\frac{1}{2} \Delta \psi(t, x)+V^{\star}(x) \psi(t, x), \quad x \in \mathbb{R}^{N}, t>0, \\
\left.\psi(t, x)\right|_{t=0}=\psi_{0}
\end{array}\right.
$$

where we assume that $\psi_{0}^{\varepsilon} \rightarrow \psi_{0}$ as $\varepsilon \rightarrow 0$ strongly in $H^{1}\left(\mathbb{R}^{N}\right)$.

For the wave function $\psi^{\varepsilon}(t, x)$ one can associate two basic real valued densities, namely, the position density and the current density defined by

$$
\rho^{\varepsilon}(t, x)=\left|\psi^{\varepsilon}(t, x)\right|^{2}, \quad J^{\varepsilon}(t, x)=\operatorname{Im}\left(\overline{\psi^{\varepsilon}}(t, x) \nabla \psi^{\varepsilon}(t, x)\right) .
$$

These quantities satisfy the conversation law

$$
\partial_{t} \rho^{\varepsilon}+\operatorname{div}_{x} J^{\varepsilon}=0
$$

The corresponding position density and current density of the effective wave function $\psi(t, x)$ defined by

$$
\rho(t, x)=|\psi(t, x)|^{2}, \quad J(t, x)=\operatorname{Im}(\bar{\psi}(t, x) \nabla \psi(t, x)) .
$$


Due to the Bohmian mechanics offered by Bohm in 1952 as an another approach to quantum mechanics, one can define particle trajectories $X_{t}^{\varepsilon}: x \rightarrow X^{\varepsilon}(t, x) \in \mathbb{R}^{N}$ via the following differential equation

$$
\left\{\begin{array}{l}
\dot{X}^{\varepsilon}(t, x)=u^{\varepsilon}\left(t, X^{\varepsilon}(t, x)\right), \\
\left.X^{\varepsilon}(t, x)\right|_{t=0}=x, \quad x \in \mathbb{R}^{N},
\end{array}\right.
$$

where the velocity field is given by

$$
u^{\varepsilon}(t, x):=\frac{J^{\varepsilon}(t, x)}{\rho^{\varepsilon}(t, x)} .
$$

The particle trajectory $X_{t}: x \rightarrow X(t, x) \in \mathbb{R}^{N}$ corresponding to the effective wave function $\psi(t, x)$ can be defined by

$$
\left\{\begin{array}{l}
\dot{X}(t, x)=u(t, X(t, x)), \\
\left.X(t, x)\right|_{t=0}=x, \quad x \in \mathbb{R}^{N},
\end{array}\right.
$$

with the velocity field

$$
u(t, x):=\frac{J(t, x)}{\rho(t, x)} .
$$

To characterize the Bohmian mechanics more rigorously, we recall the Bohmian measure from [14] and [23].

Definition 2.1 (Bohmian measure). Let $\varepsilon>0$ and $\psi^{\varepsilon} \in H^{1}\left(\mathbb{R}^{N}\right)$ be a sequence of wave functions with corresponding densities $\rho^{\varepsilon}$ and $J^{\varepsilon}$. Then the associated Bohmian measure $\beta^{\varepsilon} \in$ $\mathcal{M}^{+}\left(\mathbb{R}_{x}^{N} \times \mathbb{R}_{p}^{N}\right)$ is given by

$$
\left\langle\beta^{\varepsilon}, \varphi\right\rangle:=\int_{\mathbb{R}^{N}} \rho^{\varepsilon}(x) \varphi\left(x, \frac{\rho^{\varepsilon}(x)}{J^{\varepsilon}(x)}\right), \quad \forall \varphi \in C_{0}\left(\mathbb{R}_{x}^{N} \times \mathbb{R}_{p}^{N}\right),
$$

where $\mathcal{M}^{+}$denotes the set of nonnegative Borel measures on phase space, $\langle\cdot, \cdot\rangle$ denotes the corresponding duality bracket between $\mathcal{M}^{+}\left(\mathbb{R}_{x}^{N} \times \mathbb{R}_{p}^{N}\right)$ and $C_{0}\left(\mathbb{R}_{x}^{N} \times \mathbb{R}_{p}^{N}\right)$, and $C_{0}$ is the closure (with respect to the uniform norm) of the set of continuous functions with compact support.

We also recall the following assertions in [23] which ensures existence of a classical limit of $\beta^{\varepsilon}$.

Lemma $2.2([23])$. Let $\psi^{\varepsilon}$ be uniformly bounded in $L^{2}\left(\mathbb{R}^{N}\right)$. Then, up to extraction of sub-sequences, there exists a limiting measure $\beta \in \mathcal{M}^{+}\left(\mathbb{R}_{x}^{N} \times \mathbb{R}_{p}^{N}\right)$, such that

$$
\beta^{\varepsilon} \rightarrow \beta \quad \text { as } \quad \varepsilon \rightarrow 0_{+}
$$

weakly-» in $\mathcal{M}^{+}\left(\mathbb{R}_{x}^{N} \times \mathbb{R}_{p}^{N}\right)$.

Theorem $2.3([23])$. Let $\psi^{\varepsilon}$ be uniformly bounded in $H^{1}\left(\mathbb{R}^{N}\right)$ with corresponding densities $\rho^{\varepsilon}, J^{\varepsilon} \in L^{1}\left(\mathbb{R}^{N}\right)$. If $\rho^{\varepsilon} \stackrel{\varepsilon \rightarrow 0_{+}}{\longrightarrow} \rho$ in $L^{1}\left(\mathbb{R}^{N}\right)$ strongly and $J^{\varepsilon} \stackrel{\varepsilon \rightarrow 0_{+}}{\longrightarrow} \tilde{J}$ in measure, then $\beta$ is mono-kinetic, i.e.

$$
\beta(x, p)=\rho(t, x) \delta\left(p-\frac{\tilde{J}(t, x)}{\rho(t, x)}\right) .
$$

Finally, we collect some basic fact from measure theory and homogenization theory. 
Theorem 2.4 (Weak limits of rapidly oscillating periodic functions [7]). Let $1 \leq p \leq+\infty$ and $f$ be a $Y$-periodic function in $L^{p}(Y)$. Set

$$
f_{\varepsilon}(x)=f\left(\frac{x}{\varepsilon}\right) \quad \text { a.e. on } \mathbb{R}^{N} .
$$

Then, if $p<+\infty$, as $\varepsilon \rightarrow 0$

$$
f_{\varepsilon} \rightarrow \mathcal{M}_{Y}(f)=\frac{1}{|Y|} \int_{Y} f(y) \mathrm{d} y \quad \text { weakly in } L^{p}(\omega)
$$

for every bounded open subset $\omega$ of $\mathbb{R}^{N}$.

If $p=+\infty$, one has

$$
f_{\varepsilon} \rightarrow \mathcal{M}_{Y}(f)=\frac{1}{|Y|} \int_{Y} f(y) \mathrm{d} y \quad \text { weakly }-\star \text { in } L^{\infty}\left(\mathbb{R}^{N}\right) .
$$

The following theorem shows the relationship between some sequence and the Young measure generated by it.

Theorem 2.5 ([15]). If $|\Omega|<\infty$ and $\nu_{x}$ is the Young measure generated by the (whole) sequence $\left\{u_{j}\right\}_{j \in \mathbb{N}}$ then

$$
u_{j} \rightarrow u \text { as } j \rightarrow \infty \text { in measure } \Leftrightarrow \nu_{x}=\delta_{u(x)} \text { for a.e. } x \in \Omega \text {. }
$$

\section{Convergence of Bohmian Trajectories}

In this section, we start with the existence and boundedness of solutions of the timeoscillating Schrödinger equation (2.1) and its effective system (2.3). The results can be obtained by Galerkin approximation method and we omit the details here ([22]).

Lemma 3.1. Let the potential $V(t, x) \in C_{0}^{\infty}\left(\mathbb{R} \times \mathbb{R}^{N} ; \mathbb{R}\right)$ with period 1 is assumed to be bounded below and subquadratic, then there exists constant $T_{0}>0$ such that the wave function $\psi^{\varepsilon}(t, x)$ of (2.1) is uniformly bounded in $H^{1}\left(\mathbb{R}^{N}\right)$ for all $t \in\left[0, T_{0}\right)$.

Lemma 3.2. Let the potential $V(t, x) \in C_{0}^{\infty}\left(\mathbb{R} \times \mathbb{R}^{N} ; \mathbb{R}\right)$ with period 1 is assumed to be bounded below and subquadratic, and $V^{\star}(x)$ be as in Sect. 2, then there exists constant $T_{0}^{\star}>0$ such that he wave function $\psi(t, x)$ of $(2.3)$ is uniformly bounded in $H^{1}\left(\mathbb{R}^{N}\right)$ for all $t \in\left[0, T_{0}^{\star}\right)$.

Note that by use of theorem of weak limits of rapidly oscillating periodic functions (Theorem 2.4) presented in [7, we obtain the following theorem for the homogenized problem (2.1).

Theorem 3.3 (Convergence of wave function). Assume that the potential function satisfies (2.2) and the initial wave function $\psi_{0}^{\varepsilon}$ is strongly convergent to $\psi_{0}$ in $H^{1}\left(\mathbb{R}^{N}\right)$. Let $\hat{T}=$ $\min \left\{T_{0}, T_{0}^{\star}\right\}, \psi^{\varepsilon}(t, x)$ and $\psi(t, x)$ be the solutions for (2.1) and (2.3) respectively. Then

$$
\lim _{\varepsilon \rightarrow 0}\left\|\psi^{\varepsilon}(t, x)-\psi(t, x)\right\|_{H^{1}\left(\mathbb{R}^{N}\right)}=0 \quad \text { for all } t \in\left[0, \hat{T}^{\top}\right) .
$$

Proof. Consider the difference of $\psi^{\varepsilon}(t, x)-\psi(t, x)$, which satisfies the following equation

$$
i \frac{\partial\left(\psi^{\varepsilon}(t, x)-\psi(t, x)\right)}{\partial_{t}}=-\frac{1}{2} \Delta\left(\psi^{\varepsilon}(t, x)-\psi(t, x)\right)+V\left(\frac{t}{\varepsilon}, x\right) \psi^{\varepsilon}(t, x)-V^{\star}(x) \psi(t, x) .
$$


Multiplying both sides of (3.1) by $-\Delta \overline{\left(\psi^{\varepsilon}(t, x)-\psi(t, x)\right)}$, then

$$
\begin{aligned}
& i a\left(\frac{\partial\left(\psi^{\varepsilon}(t, x)-\psi(t, x)\right)}{\partial_{t}}, \psi^{\varepsilon}(t, x)-\psi(t, x)\right) \\
= & \left.\frac{1}{2}\left(\Delta\left(\psi^{\varepsilon}(t, x)-\psi(t, x)\right), \Delta \overline{\left(\psi^{\varepsilon}(t, x)-\psi(t, x)\right)}\right)\right)-\left(V\left(\frac{t}{\varepsilon}, x\right) \psi^{\varepsilon}(t, x), \Delta \overline{\left(\psi^{\varepsilon}(t, x)-\psi(t, x)\right)}\right) \\
& +V^{\star}(x) a\left(\psi^{\varepsilon}(t, x)-\psi(t, x), \psi^{\varepsilon}(t, x)-\psi(t, x)\right),
\end{aligned}
$$

where

$$
a(\psi(x), \phi(x))=\sum_{i=1}^{N} \int_{\mathbb{R}^{\mathbb{N}}} \frac{\partial \psi(x)}{\partial x_{i}} \cdot \frac{\partial \phi(x)}{\partial x_{i}} \mathrm{~d} x
$$

for any $\psi(x), \phi(x) \in H^{1}\left(\mathbb{R}^{N}\right)$. Taking the imaginary part of (3.2), we have

$$
\begin{array}{r}
\frac{\mathrm{d}}{\mathrm{d} t}\left\|\psi^{\varepsilon}(t, x)-\psi(t, x)\right\|_{H^{1}\left(\mathbb{R}^{N}\right)}^{2} \leq\left|\left(V\left(\frac{t}{\varepsilon}, x\right)-V^{\star}(x), \psi^{\varepsilon}(t, x) \Delta\left(\psi^{\varepsilon}(t, x)-\psi(t, x)\right)\right)\right| \\
+M\left\|\psi^{\varepsilon}(t, x)-\psi(t, x)\right\|_{H^{1}\left(\mathbb{R}^{N}\right)}^{2},
\end{array}
$$

where $M:=\|V(t, x)\|_{L^{\infty}}$ and $M$ is finite since the assumption that $V(t, x) \in C_{0}^{\infty}\left(\mathbb{R} \times \mathbb{R}^{N} ; \mathbb{R}\right)$.

Then for any $t \in[0, \hat{T})$, the following inequality can be obtained by applying the Gronwall inequality

$$
\left\|\psi^{\varepsilon}(t, x)-\psi(t, x)\right\|_{H^{1}\left(\mathbb{R}^{N}\right)}^{2} \leq\left(\left\|\psi_{0}^{\varepsilon}-\psi_{0}\right\|_{H^{1}\left(\mathbb{R}^{N}\right)}^{2}+\int_{0}^{t} e^{(t-s) M} b^{\varepsilon}(s) \mathrm{d} s\right),
$$

where the constant $C>0$ independent of $t, x, \varepsilon$ and

$$
b^{\varepsilon}(t):=\left|\left(V\left(\frac{t}{\varepsilon}, x\right)-V^{\star}(x), \psi^{\varepsilon}(t, x) \Delta\left(\psi^{\varepsilon}(t, x)-\psi(t, x)\right)\right)\right| .
$$

For the rapidly oscillating periodic potential, Theorem 2.4 implies that

$$
V\left(\frac{t}{\varepsilon}, x\right) \stackrel{\varepsilon \rightarrow 0}{\longrightarrow} V^{\star}(x) \quad \text { weakly }-\star \text { in } L^{\infty}\left(\mathbb{R}^{N}\right) .
$$

Then together with the fact that $\psi^{\varepsilon}(t, x) \Delta\left(\psi^{\varepsilon}(t, x)-\psi(t, x)\right) \in L^{1}\left(\mathbb{R}^{N}\right)$, we can further deduce that

$$
b^{\varepsilon}(t)=\left|\left(V\left(\frac{t}{\varepsilon}, x\right)-V^{\star}(x), \psi^{\varepsilon}(t, x) \Delta\left(\psi^{\varepsilon}(t, x)-\psi(t, x)\right)\right)\right| \stackrel{\varepsilon \rightarrow 0}{\longrightarrow} 0 .
$$

Moreover the fact that $\psi^{\varepsilon}(t, x) \Delta\left(\psi^{\varepsilon}(t, x)-\psi(t, x)\right) \in L^{1}\left(\mathbb{R}^{N}\right)$ follows from that

$$
\begin{aligned}
& \int_{\mathbb{R}^{N}}\left|\psi^{\varepsilon}(t, x) \Delta\left(\psi^{\varepsilon}(t, x)-\psi(t, x)\right)\right| \mathrm{d} x \\
\leq & \left\|\psi^{\varepsilon}(t, x)\right\|_{H^{1}\left(\mathbb{R}^{N}\right)} \cdot\left\|\Delta\left(\psi^{\varepsilon}(t, x)-\psi(t, x)\right)\right\|_{H^{-1}\left(\mathbb{R}^{N}\right)} \\
< & \infty,
\end{aligned}
$$

where the last inequality holds because of Lemma 3.1 and 3.2 .

Thus together with (3.5) and the assumption that $\psi_{0}^{\varepsilon \stackrel{\varepsilon \rightarrow 0}{\longrightarrow}} \psi_{0}$ strongly in $H^{1}\left(\mathbb{R}^{N}\right)$, we conclude from (3.4) that

$$
\left\|\psi^{\varepsilon}(t, x)-\psi(t, x)\right\|_{H^{1}\left(\mathbb{R}^{N}\right)}^{2} \stackrel{\varepsilon \rightarrow 0}{\longrightarrow} 0 \quad \text { for all } t \in[0, \hat{T}) .
$$

This proof of Theorem 3.3 is complete. 
The following Theorem is about the convergence of the position density $\rho^{\varepsilon}$ and the current density $J^{\varepsilon}$. This is a natural consequence of Theorem 3.3 .

Theorem 3.4 (Convergence of densities). Assume that the assumptions in Theorem 3.3 hold. Let $\hat{T}$ be as in Theorem [3.3, $\psi^{\varepsilon}(t, x)$ and $\psi(t, x)$ be the solutions for (2.1) and (2.3) respectively, then

$$
\rho^{\varepsilon} \stackrel{\varepsilon \rightarrow 0_{+}}{\longrightarrow} \rho \text { in } L^{1}\left(\mathbb{R}^{N}\right) \text { strongly for all } t \in\left[0, \hat{T}^{\top}\right),
$$

and

$$
J^{\varepsilon} \stackrel{\varepsilon \rightarrow 0_{+}}{\longrightarrow} J \text { in } L^{1}\left(\mathbb{R}^{N}\right) \text { strongly for all } t \in\left[0, \hat{T}^{\top}\right) .
$$

Proof. The uniform boundness of $\psi^{\varepsilon}$ in Lemma 3.1 implies that the corresponding densities $\rho^{\varepsilon}(t, x), J^{\varepsilon}(t, x) \in L^{1}\left(\mathbb{R}^{N}\right)$, and so do $\rho(t, x)$ and $J(t, x)$.

With the definition of $\rho^{\varepsilon}(t, x)$ and $\rho(t, x)$ in hand, we can show that

$$
\begin{aligned}
& \int_{\mathbb{R}^{N}}\left|\rho^{\varepsilon}(t, x)-\rho(t, x)\right| \mathrm{d} x \\
= & \left.\int_{\mathbb{R}^{N}}|| \psi^{\varepsilon}(t, x)\right|^{2}-|\psi(t, x)|^{2} \mid \mathrm{d} x \\
= & \int_{\mathbb{R}^{N}}\left|\psi^{\varepsilon}(t, x) \overline{\psi^{\varepsilon}}(t, x)-\psi(t, x) \bar{\psi}(t, x)\right| \mathrm{d} x \\
\leq & \int_{\mathbb{R}^{N}}\left|\overline{\psi^{\varepsilon}}(t, x)\left\|\psi^{\varepsilon}(t, x)-\psi(t, x)\left|\mathrm{d} x+\int_{\mathbb{R}^{N}}\right| \psi(t, x)\right\| \overline{\psi^{\varepsilon}}(t, x)-\bar{\psi}(t, x)\right| \mathrm{d} x \\
\leq & \left\|\psi^{\varepsilon}(t, x)\right\|_{L^{2}\left(\mathbb{R}^{N}\right)}\left\|\psi^{\varepsilon}(t, x)-\psi(t, x)\right\|_{L^{2}\left(\mathbb{R}^{N}\right)}+\|\psi(t, x)\|_{L^{2}\left(\mathbb{R}^{N}\right)}\left\|\psi^{\varepsilon}(t, x)-\psi(t, x)\right\|_{L^{2}\left(\mathbb{R}^{N}\right)} \\
& \rightarrow 0 \quad \text { as } \varepsilon \rightarrow 0,
\end{aligned}
$$

where the last assertion follows from the uniform boundness of $\psi^{\varepsilon}, \psi$ in Lemma 3.1 and Lemma 3.2 and convergence of $\psi^{\varepsilon}$ in Theorem 3.3 .

Also the definition of the current densities $J^{\varepsilon}(t, x)$ and $J(t, x)$ imply that

$$
\begin{aligned}
& \int_{\mathbb{R}^{N}}\left|J^{\varepsilon}(t, x)-J(t, x)\right| \mathrm{d} x \\
= & \int_{\mathbb{R}^{N}}\left|\operatorname{Im}\left(\overline{\psi^{\varepsilon}}(t, x) \nabla \psi^{\varepsilon}(t, x)\right)-\operatorname{Im}(\bar{\psi}(t, x) \nabla \psi(t, x))\right| \mathrm{d} x \\
\leq & \left\|\psi^{\varepsilon}(t, x)\right\|_{L^{2}\left(\mathbb{R}^{N}\right)}\left\|\nabla \psi^{\varepsilon}(t, x)-\nabla \psi(t, x)\right\|_{L^{2}\left(\mathbb{R}^{N}\right)}+\|\nabla \psi(t, x)\|_{L^{2}\left(\mathbb{R}^{N}\right)}\left\|\psi^{\varepsilon}(t, x)-\psi(t, x)\right\|_{L^{2}\left(\mathbb{R}^{N}\right)} \\
& \rightarrow 0 \quad \text { as } \varepsilon \rightarrow 0,
\end{aligned}
$$

where the last assertion follows from the uniform boundness of $\psi^{\varepsilon}, \psi$ in Lemma 3.1 and Lemma 3.2 and convergence of $\psi^{\varepsilon}$ in Theorem 3.3. The proof of Theorem 3.4 is complete.

Theorem 3.5 (Convergence of Bohmian measures). Assume that the assumptions in Theorem 3.3 hold. Let $\psi^{\varepsilon}(t, x)$ and $\psi(t, x)$ be the solutions for (2.1) and (2.3) respectively, then up to extraction of sub-sequences, there exists a limiting measure $\beta \in \mathcal{M}^{+}\left(\mathbb{R}_{x}^{N} \times \mathbb{R}_{p}^{N}\right)$, such that

$$
\beta^{\varepsilon} \rightarrow \beta \quad \text { as } \quad \varepsilon \rightarrow 0_{+}
$$

weakly- $\star$ in $\mathcal{M}^{+}\left(\mathbb{R}_{x}^{N} \times \mathbb{R}_{p}^{N}\right)$ and the limit $\beta$ is mono-kinetic, i.e.

$$
\beta(x, p)=\rho(t, x) \delta\left(p-\frac{J(t, x)}{\rho(t, x)}\right) .
$$


Proof. Since $\psi^{\varepsilon}$ is uniformly bounded in $H^{1}\left(\mathbb{R}^{N}\right)$ in Lemma 3.1, then by Lemma 2.2 there exists some measure $\beta$ such that

$$
\beta^{\varepsilon} \rightarrow \beta \quad \text { as } \quad \varepsilon \rightarrow 0_{+}
$$

weakly- $\star$ in $\mathcal{M}^{+}\left(\mathbb{R}_{x}^{N} \times \mathbb{R}_{p}^{N}\right)$. Then due to the Theorem 2.3 and the strong convergence of $\rho^{\varepsilon}(t, x)$ and $J^{\varepsilon}(t, x)$ stated in Theorem 3.4, we can infer that the limiting measure is is mono-kinetic and given by

$$
\beta(x, p)=\rho(t, x) \delta\left(p-\frac{J(t, x)}{\rho(t, x)}\right) .
$$

The proof of Theorem 3.5 is complete.

Now we reformulate the Bohmian mechanics in its Lagrangian formulation([14, 23]). Let the motion be $P^{\varepsilon}=u^{\varepsilon}\left(x, X^{\varepsilon}(t, x)\right)$, we can differentiate $P^{\varepsilon}(t, x) \rho_{0}^{\varepsilon}$-a.e. to obtain

$$
\dot{P}^{\varepsilon}(t, x)=\partial_{t} u^{\varepsilon}\left(x, X^{\varepsilon}(t, x)\right)+\left(u^{\varepsilon}\left(x, X^{\varepsilon}(t, x)\right) \cdot \nabla\right) u^{\varepsilon}\left(x, X^{\varepsilon}(t, x)\right) .
$$

With assumptions given on the potential $V(t, x)$, we can infer that the wave function $\psi^{\varepsilon}(t, x)$ is smooth enough([15]). Then one can deduce a closed system of equations for the densities $\rho^{\varepsilon}$ and $J^{\varepsilon}$ from the oscillating Schrödinger equations (2.1), i.e. the well-known hydrodynamic formulation of quantum mechanics ([31]) which holds in the sense of distribution

$$
\left\{\begin{array}{l}
\partial_{t} \rho^{\varepsilon}+\operatorname{div} J^{\varepsilon}=0, \\
\partial_{t} J^{\varepsilon}+\operatorname{div}\left(\frac{J^{\varepsilon} \otimes J^{\varepsilon}}{\rho^{\varepsilon}}\right)+\rho^{\varepsilon} \nabla V=\frac{1}{2} \rho^{\varepsilon} \nabla\left(\frac{\Delta \sqrt{\rho^{\varepsilon}}}{\sqrt{\rho^{\varepsilon}}}\right) .
\end{array}\right.
$$

Note that $J^{\varepsilon}=\rho^{\varepsilon} u^{\varepsilon}$, together with (3.7) we can formulate the following system of ordinary differential equations which fully determines the quantum mechanical dynamics

$$
\left\{\begin{array}{l}
\dot{X}^{\varepsilon}=P^{\varepsilon} \\
\dot{P}^{\varepsilon}=-\nabla V\left(\frac{t}{\varepsilon}, X^{\varepsilon}\right)+\frac{1}{2} \frac{\Delta \sqrt{\rho^{\varepsilon}}}{\sqrt{\rho^{\varepsilon}}}, \\
\left.X^{\varepsilon}(t, x)\right|_{t=0}=x \\
\left.P^{\varepsilon}(t, x)\right|_{t=0}=u^{\varepsilon}(0, x),
\end{array}\right.
$$

where the initial velocity given by

$$
u^{\varepsilon}(0, x):=\frac{J_{0}^{\varepsilon}}{\rho_{0}^{\varepsilon}}=\frac{\operatorname{Im}\left(\psi_{0}^{\varepsilon} \nabla \psi_{0}^{\varepsilon}\right)}{\left|\psi_{0}^{\varepsilon}\right|^{2}}
$$

Theorem 2.5implies that the limit of Bohmian trajectories is connected to the Young measure generated by the Bohmian dynamics. To proceed further we recall the following definition of the Young measure. For the solutions $\Phi^{\varepsilon}(t, y):=\left(X^{\varepsilon}(t, y), P^{\varepsilon}(t, y)\right)$ which is measurable in $t$ and $y$, there exists an associated Young measure $(t, y) \rightarrow \Upsilon_{t, y}(d x, d p)([2$, 17, 14] $)$

$$
\Upsilon_{t, y}: \mathbb{R}_{t} \times \mathbb{R}_{y}^{N} \rightarrow \mathcal{M}^{+}\left(\mathbb{R}_{y}^{N} \times \mathbb{R}_{p}^{N}\right),
$$

where the Young measure is defined through the following limit: for any test function $\varphi \in$ $L^{1}\left(\mathbb{R}_{t} \times \mathbb{R}_{y}^{N} ; C_{0}\left(\mathbb{R}^{2 N}\right)\right)$,

$$
\lim _{\varepsilon \rightarrow 0} \iint_{\mathbb{R} \times \mathbb{R}^{N}} \varphi\left(t, y, \Phi^{\varepsilon}(t, y)\right) \mathrm{d} y \mathrm{~d} t=\iint_{\mathbb{R} \times \mathbb{R}^{N}} \iint_{\mathbb{R}^{2 N}} \varphi(t, y, x, p) \Upsilon_{t, y}(d x, d p) \mathrm{d} y \mathrm{~d} t .
$$


Let us draw the conclusion on the convergence of Bohmian trajectories (3.8) through the connection between the limiting Bohmian measure $\beta$ and the Young measure $\Upsilon_{t, y}(t, y)$. From [17], the Bohmian flow $x \rightarrow X^{\varepsilon}(t, x)$ is well-defined $\rho_{0}^{\varepsilon}$-a.e. for all $t \in \mathbb{R}^{+}$regardless of the continuity of $u^{\varepsilon}$ and $\rho_{0}^{\varepsilon}:=\left|\psi_{0}^{\varepsilon}\right|^{2}$. The assertion also holds for the Bohmian flow $x \rightarrow X(t, x)$ with measure $\rho_{0}:=\left|\psi_{0}\right|^{2}$. Note that $u(t, x)$ is not Lipschitz continuous for all $t \in \mathbb{R}^{+}$, there exists some constant $T^{\star}>0$ ( $T^{\star}$ could be very small) such that the Bohmian flow $X(t, x)$ is one-to-one for $t \in\left[0, T^{\star}\right)([16])$.

Theorem 3.6 (Convergence of Bohmian trajectories). Under the assumptions that the potential function satisfies (2.2) and the initial wave functions $\psi_{0}^{\varepsilon}$ is strongly convergence in $H^{1}\left(\mathbb{R}^{N}\right)$, then for all compact time intervals $I \subset[0, T)$ and $T=\min \left\{T_{0}, T_{0}^{\star}, T^{\star}\right\}$, the following convergence of corresponding Bohmian trajectories and momentum of (3.8) hold locally in measure $\rho_{0}$ on $\left\{I \times \operatorname{supp} \rho_{0}\right\} \subset \mathbb{R} \times \mathbb{R}^{N}$

$$
X^{\varepsilon} \stackrel{\varepsilon \rightarrow 0_{+}}{\longrightarrow} X, \quad P^{\varepsilon \stackrel{\varepsilon \rightarrow 0_{+}}{\longrightarrow}} P .
$$

Furthermore, the limit position-momentum pair $(X, P)$ is the solution of

$$
\left\{\begin{array}{l}
\dot{X}=P \\
\dot{P}=-\nabla V^{\star}(X)+\frac{1}{2} \frac{\Delta \sqrt{\rho}}{\sqrt{\rho}} \\
P(t, x)=u(t, X(t, x)) \\
\left.X(t, x)\right|_{t=0}=x \quad \text { and }\left.\quad P(t, x)\right|_{t=0}=u(0, x) .
\end{array}\right.
$$

More precisely, for every $\delta>0$ and every Borel set $\Omega \subset\left\{I \times \operatorname{supp} \rho_{0}\right\}$ with finite Lebesgue measure $\mathscr{L}^{N+1}$, it holds that

$$
\lim _{\varepsilon \rightarrow 0} \mathscr{L}^{N+1}\left(\left\{(t, y) \in \Omega:\left|\left(X^{\varepsilon}(t, y), P^{\varepsilon}(t, y)\right)-(X(t, y), P(t, y))\right| \geq \delta\right\}\right)=0 .
$$

Proof. The assumption that $\psi_{0}^{\varepsilon \stackrel{\varepsilon \rightarrow 0}{\longrightarrow}} \psi_{0}$ strongly in $H^{1}\left(\mathbb{R}^{N}\right)$ implies that

$$
\rho_{0}^{\varepsilon} \stackrel{\varepsilon \rightarrow 0_{+}}{\longrightarrow} \rho_{0} \quad \text { strongly in } L_{+}^{1}\left(\mathbb{R}^{N}\right) .
$$

Then the following assertion can be obtained from [23] together with (3.10)

$$
\beta(t, x, p)=\int_{\mathbb{R}_{y}^{N}} \Upsilon_{t, y}(t, p) \rho_{0}(y) \mathrm{d} y .
$$

With identity (3.11) and the convergence of Bohmian measures (Theorem 3.5) in mind, we can infer that the Young measure $\Upsilon_{t, y}(t, p)$ is supported in a single point by analogous reasonings as in [14]. That is the Young measure $\Upsilon_{t, y}(t, p)$ can be given by

$$
\Upsilon_{t, y}(t, p)=\delta(x-X(x, y)) \delta(p-u(t, X(t, y))) \quad \text { a.e. on } \operatorname{supp} \rho_{0} \subset \mathbb{R}^{N} .
$$

Thus the following assertion holds from Theorem 2.5] in measure theory

$$
X^{\varepsilon} \stackrel{\varepsilon \rightarrow 0_{+}}{\longrightarrow} X, \quad P^{\varepsilon} \stackrel{\varepsilon \rightarrow 0_{+}}{\longrightarrow} P,
$$

locally in measure on $\left\{I \times \operatorname{supp} \rho_{0}\right\} \subset \mathbb{R} \times \mathbb{R}^{N}$. The proof of Theorem 3.6 is complete. 


\section{Conclusion and Discussion}

In this paper, we have studied the limit of the Bohmian trajectory under assumptions on the potential and on the considered class of initial wave function $\psi_{0}^{\varepsilon}$. The Bohmian trajectories we considered are guided by a family of wave functions which satisfied the Schrödinger equations with rapidly oscillating potential. Due to the complicated relation between wave functions and the Bohmian trajectories, the convergence of the Schrödinger equations does not directly imply convergence of the corresponding Bohmian trajectories. In order to gain more insight, we recalled the Bohmian measure which naturally arise in the Bohmian interpretation of quantum mechanics and studied the limit of these measures. On this foundation, together with a wellknown theorem in measure theory for Young measure corresponding to the Bohmian flow, we managed to deduce that the Bohmian trajectories converge locally in measure. However, the conclusion holds when the Bohmian trajectory is one-to-one on finite time interval as a result of the lack of continuity of velocity field. We also have several comments in the following.

1. The study in this paper is important to understand how the time-oscillating Schrödinger equations affect the corresponding Bohmian trajectories in some finite time interval. Our approach can also be applied to a class of Schrödinger equations when the potential has other heterogeneities ([4, 7]). With techniques provided in homogenization ([10]), we can study the Bohmian trajectory guided by the effective Schrödinger equation. This can be taken as a simplified method in the study of Bohmian mechanics.

2. We only studied the Schrödinger equation with linear potential. It would be interesting and useful to consider the Bohmian trajectories guided by the Schrödinger equation with nonlinear potential, e.g. $V(\cdot)=g\left(|\psi|^{2}\right)$. The main obstacle at this point is the existence of Bohmian trajectories. It is conceivable that the results in this paper could be generalized with suitable assumptions on the initial wave function and the nonlinear potential.

3. Chaos in quantum mechanics is a problem of great current interest. Bohmian trajectories that approach the nodal point are chaotic in general, and the Bohmian trajectories could be chaotic if the classical potential is time dependent in more than one-dimensional system $([8,9,12,18,29,130])$. In this paper, the convergence of Bohmian trajectory is valid in a finite time interval where the Bohmian flow is one-to-one and chaos has not occurred in this time interval. However, when the Bohmian trajectory is chaotic, whether the Bohmian trajectory guided by the time-oscillating Schrödinger equation has a limit, what is the

expression of the limit and how the convergence happens are still unknown and worthy of study.

\section{Acknowledgement}

We would like to thank Stephen Wiggins for bringing us to the study of Bohmian mechanics.

\section{Data Availability}

The data that support the findings of this study are available from the corresponding author upon reasonable request. 


\section{References}

[1] G. Allaire and A. Piatnitski. Homogenization of the Schrödinger equation and effective mass theorems. Comm. Math. Phys. 258 (2005), no. 1, 1-22.

[2] J. M. Ball. A version of the fundamental theorem for Young measures. PDEs and continuum models of phase transitions (Nice, 1988), 207-215, Lecture Notes in Phys., 344, Springer, Berlin, 1989.

[3] A. Benseny, G. Albareda, Á. S. Sanz, et al. Applied bohmian mechanics. Eur. Phys. J. D, 2014, 68(10): 1-42.

[4] A. Bensoussan, J. L. Lions and Papanicolaou G. Asymptotic analysis for periodic structures. American Mathematical Soc., 2011.

[5] A. Bishop, R. Flesch, M. Forest, D. McLaughlin and E. A. Overman. Correlations between chaos in a perturbed sine-Gordon equation and a truncated model system. SIAM J. Math. Anal. 21 (1990), no. 6, 1511-1536.

[6] D. Bohm. A suggested interpretation of the quantum theory in terms of "hidden" variables. I. Phys. Rev. (2) 85 (1952), 166-179.

[7] D. Cioranescu and P. Donato. An introduction to homogenization. Oxford: Oxford University Press, 1999.

[8] G. Contopoulos and C. Efthymiopoulos. Ordered and chaotic Bohmian trajectories. Celestial Mech. Dynam. Astronom. 102 (2008), no. 1-3, 219-239.

[9] G. Contopoulos and A. Tzemos. Chaos in Bohmian Quantum Mechanics: A Short Review. Regul. Chaotic Dyn. 25 (2020), no. 5, 476-495.

[10] J. Duan and W. Wang. Effective dynamics of stochastic partial differential equations. Elsevier Insights. Elsevier, Amsterdam, 2014.

[11] D. Dürr and S. Römer. On the classical limit of Bohmian mechanics for Hagedorn wave packets. J. Funct. Anal. 259 (2010), no. 9, 2404-2423.

[12] C. Efthymiopoulos and G. Contopoulos. Chaos in Bohmian quantum mechanics. J. Phys. A. 39(2006), no. 8, 1819-1852.

[13] E. Feireisl and A. Novotný. Singular limits in thermodynamics of viscous fluids. Advances in Mathematical Fluid Mechanics. Birkhäuser Verlag, Basel, 2009.

[14] A. Figalli, C. Klein, P. Markowich and C. Sparber. WKB analysis of Bohmian dynamics. Comm. Pure Appl. Math. 67 (2014), no. 4, 581-620.

[15] I. Gasser and P. Markowich. Quantum hydrodynamics, Wigner transforms and the classical limit. Asymptot. Anal. 14 (1997), no. 2, 97-116.

[16] L. Hörmander. Symplectic classification of quadratic forms, and general Mehler formulas. Math. Z. 219 (1995), no. 3, 413-449.

[17] N. Hungerbühler. A refinement of Ball's theorem on Young measures. New York J. Math. 3 (1997), 48-53. 
[18] G. Iacomelli and M. Pettini. Regular and chaotic quantum motions. Phys. Lett. A. 212 (1996), no. 1-2, 29-38.

[19] S. Klainerman and A. Majda. Singular limits of quasilinear hyperbolic systems with large parameters and the incompressible limit of compressible fluids. Comm. Pure Appl. Math. 34 (1981), no. 4, 481-524.

[20] D. Li. Asymptotics of singularly perturbed damped wave equations with super-cubic exponent. Discrete Contin. Dyn. Syst. Ser. B, 2021. DOI: 10.3934/dcdsb.2021056.

[21] L. Lin, M. Yang and J. Duan. Effective Approximation for a Nonlocal Stochastic Schrö dinger Equation with Oscillating Potential. arXiv preprint arXiv:1906.09375, 2019.

[22] J. L. Lions and E. Magenes. Non-homogeneous boundary value problems and applications: Vol. 1. Springer Science and Business Media, 2012.

[23] P. Markowich, T. Paul and C. Sparber. Bohmian measures and their classical limit. J. Funct. Anal. 259 (2010), no. 6, 1542-1576.

[24] A. Sanz and S. Miret-Artés. A trajectory description of quantum processes. II. Applications. A Bohmian perspective. Lecture Notes in Physics, 831. Springer, Heidelberg, 2014.

[25] L. Signing. Periodic homogenization of Schrödinger type equations with rapidly oscillating potential. Afr. Diaspora J. Math. 19 (2016), no. 2, 29-45.

[26] C. Sparber. Semiclassical Quantum Dynamics and Bohmian trajectories. Notices of the AMS, 2016, 63(9).

[27] S. Teufel and D. Dürr. Bohmian Mechanics: The Physics and Mathematics of Quantum Theory. Springer-Verlag Berlin Heidelberg, 2009.

[28] S. Teufel and R. Tumulka. Simple proof for global existence of Bohmian trajectories. Comm. Math. Phys. 258 (2005), no. 2, 349-365.

[29] D. A. Wisniacki and E. R. Pujals. Motion of vortices implies chaos in Bohmian mechanics. Europhys. Lett. 71 (2005), no. 2, 159-165.

[30] D. A. Wisniacki, E. R. Pujals and F. Borondo. Vortex interaction, chaos and quantum probabilities. Europhys. Lett. 73 (2006), no. 5, 671-676.

[31] R. E. Wyatt. Quantum dynamics with trajectories: introduction to quantum hydrodynamics. Springer Science and Business Media, 2006. 